\title{
Elastic nucleon-deuteron scattering and breakup with chiral forces
}

\author{
Henryk Witąa, Jacek Golak, Roman Skibiński and \\ KACPER TOPOLNICKI
}

Institute of Physics, Jagiellonian University, PL-30348 Kraków

\begin{abstract}
Results on three-nucleon (3N) elastic scattering and breakup below the pion production threshold are discussed. The large discrepancies found between a theory based on numerical solutions of 3N Faddeev equations with standard nucleon-nucleon (NN) potentials only and data point to the need for three-nucleon forces (3NF's). This notion is supported by the fact that another possible reason for the discrepancies in elastic nucleon-deuteron ( $\mathrm{Nd}$ ) scattering, relativistic effects, turned out to be small. Results for a new generation of chiral NN forces (up to $\mathrm{N}^{4} \mathrm{LO}$ ) together with theoretical truncation errors are shown. They support conclusions obtained with standard NN potentials
\end{abstract}

\section{Introduction}

Traditionally in nuclear physics the Hamiltonian has been taken in a nonrelativistic form in which pairwise interactions between nucleons are supplemented by 3NF's for systems with more than two nucleons. The $3 \mathrm{~N}$ system is the first nontrivial case where NN potentials can be tested. In that system also for the first time 3NF's come into play making it a valuable source of information on $3 \mathrm{NF}$ properties and their significance in the nuclear Hamiltonian.

The need for 3NF's was established when three- and four-nucleon bound states have been solved exactly using high precision semi(phenomenological) NN potentials [1-3]. It was natural to look for an explanation of resulting 
underbinding by introducing 3NF's such as the Urbana IX (UIX) [4] or the Tucson-Melbourne (TM) model [5] into the nuclear Hamiltonian.

Effective field theoretical methods in a form of chiral perturbation theory $(\chi \mathrm{PT})$ provided a solid basis for a construction of nuclear forces. Consistent chiral NN $[6,7]$ and $3 \mathrm{~N}[8,9]$ forces have been derived in the $\chi \mathrm{PT}$ framework. Recently improved chiral NN potentials have been constructed by using regularization in the coordinate space $[10,11]$. This significantly reduced finite-cutoff artefacts of the nonlocal momentum-space regulator used in $[6,7]$ allowing us to apply the improved forces to higher energy Nd scattering.

In the next section we briefly review comparison of $3 \mathrm{~N}$ scattering data to various NN potential predictions alone or combined with different 3NF's. These examples are chosen to show the importance of the $3 \mathrm{NF}$ in the $3 \mathrm{~N}$ system. The $3 \mathrm{NF}$ effects seem to grow with increasing energy of the $3 \mathrm{~N}$ system. We also discuss the importance of relativistic effects in elastic Nd scattering and their significance in the study of $3 \mathrm{NF}$ effects. The small size of relativistic effects indicates that one should focus on 3NF's, specifically of short-range nature. In the following section we present examples of results for improved chiral NN potentials. We summarize in the last section.

\section{$23 \mathrm{~N}$ reactions with standard forces}

All observables for elastic Nd scattering can be obtained from solutions of $3 \mathrm{~N}$ Faddeev equations. Presently they can be solved numerically exactly for any NN potential and 3NF [12]. Using the realistic NN forces: AV18 [1], CD Bonn [2], Nijm1, Nijm2, and Nijm93 [3] one gets, in general, predictions for $3 \mathrm{~N}$ scattering observables which agree well with data at energies below $\approx 30 \mathrm{MeV}$. A fairly complete overview of those theoretical predictions in comparison to data is presented in $[12,13]$. At higher energies discrepancies develop. They are exemplified for the neutron-deuteron (nd) elastic scattering cross section in Figure 1A. The large discrepancy in the minimum of the elastic scattering cross section obtained with NN forces only, seen for energies above $\approx 60 \mathrm{MeV}$, is removed for energies below $\approx 140 \mathrm{MeV}$ when $3 \mathrm{NF}$ 's, which reproduce the experimental triton binding energy, are included [13]. A similar behavior shows up for the high energy deuteron vector analyzing power $A_{y}(d)[13]$. But there are many spin observables for which large $3 \mathrm{NF}$ effects are predicted and where the TM and the Urbana IX do not reproduce the data [13]. This is the case e.g. for the nucleon analyzing power $A_{y}$ [13] and for the deuteron tensor analyzing powers [13]. In none of these cases the data can be reproduced by pure $2 \mathrm{~N}$ force predictions. Also for the $\mathrm{Nd}$ 


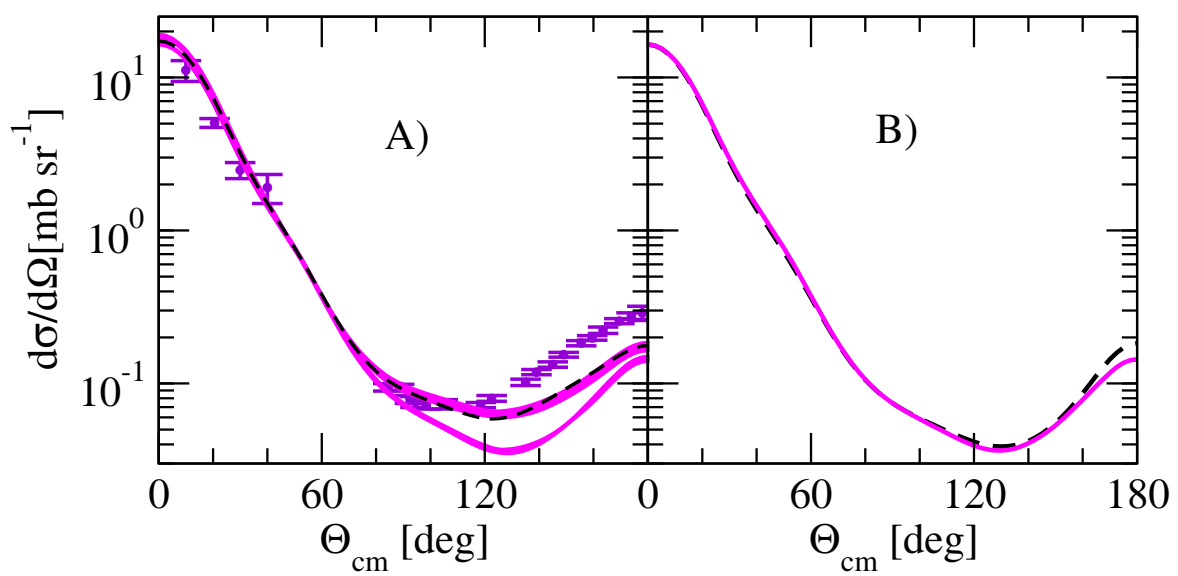

Figure 1: The angular distribution for nd elastic scattering at neutron energy $E_{l a b}=$ $250 \mathrm{MeV}$. The nd experimental data (solid dots) are from [19]. In A) the lower and upper bands result from predictions of (semi)phenomenological potentials alone (AV18, CD Bonn, Nijm1, Nijm2) and combined with TM99 3NF [20], respectively. The dashed line is the prediction of the AV18+UIX 3NF. In B) the solid and dashed lines are nonrelativistic and relativistic predictions based on the CD Bonn potential.

breakup reaction there are spin observables which require 3NF's for their explanation $[14,15]$. Therefore observables, which are sensitive to the $3 \mathrm{NF}$ structure, can be identified for elastic Nd scattering and deuteron breakup.

There are large discrepancies at energies above $\approx 140 \mathrm{MeV}$ between data and theory in elastic Nd scattering which cannot be removed by adding standard 3NF's (see Figure 1A). They require to study the magnitude of relativistic effects. We used an instant form relativistic approach which encompasses relativistic kinematics, boost corrections and Wigner spin rotations $[16,17]$. It turned out that relativistic effects lead to a rather small effects in the cross section, mostly restricted to the backward angles [16] (see Figure 1B). Higher energy elastic scattering spin observables are only slightly modified by relativity $[16,17]$.

\section{Results with chiral NN forces}

The small size of relativistic effects indicates that very likely the short range contributions to the $3 \mathrm{NF}$ are responsible for the higher energy elastic scattering discrepancies. The recently constructed new generation of chiral NN potentials up to $\mathrm{N}^{4} \mathrm{LO}$ with an appropriate regularization in the coordinate space $[10,11]$ made it possible to reduce significantly finite-cutoff artefacts 


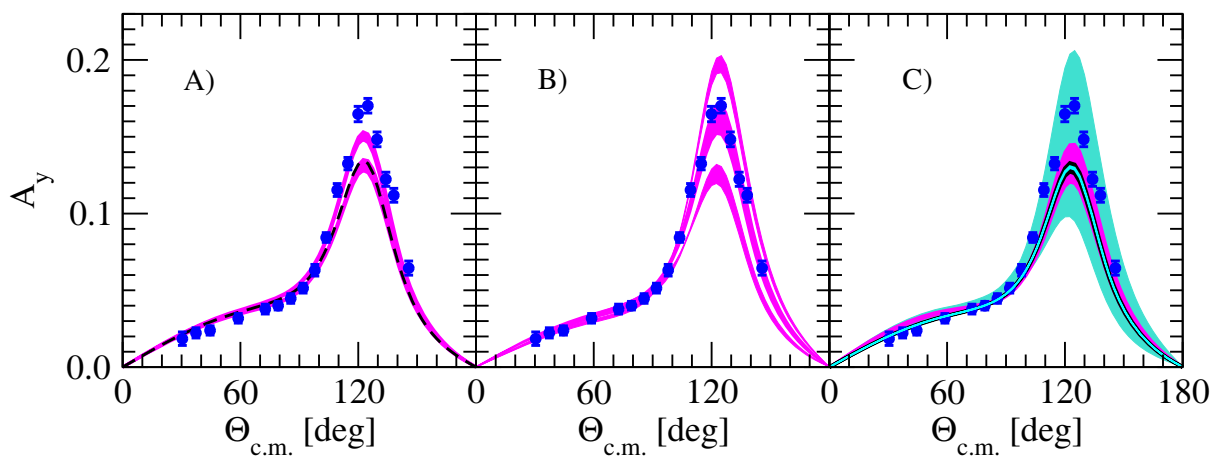

Figure 2: The nd elastic scattering analyzing power $A_{y}$ at $E_{l a b}=10 \mathrm{MeV}$. For description of bands and lines in A) see Figure 1A. In B) bands of predictions for five versions of the old Bochum chiral NN potentials of Ref. [6] at different orders of the chiral expansion are shown: NLO - the upper band, $\mathrm{N}^{2} \mathrm{LO}$ - the middle band, and $\mathrm{N}^{3} \mathrm{LO}$ - the bottom band. In $\mathrm{C}$ ) predictions based on the improved chiral NN potentials of Refs. $[10,11]$ with local regulator and parameter $R=1.0 \mathrm{fm}$ are shown. The bands of increasing width show estimated theoretical uncertainty: the narrowest at $\mathrm{N}^{4} \mathrm{LO}$, slightly wider at $\mathrm{N}^{3} \mathrm{LO}$, then $\mathrm{N}^{2} \mathrm{LO}$ and widest at NLO. The full circles are nd data from Ref. [21].

present when using the nonlocal momentum-space regulator employed in the chiral NN potentials of Refs. [6,7]. These artefacts are clearly seen in strong variations around $p=2 \mathrm{fm}^{-1}$ of the $l=0$ component of the deuteron wave function calculated with $\mathrm{N}^{3} \mathrm{LO} \mathrm{NN}$ potentials of Refs. [6] and [7]. They are absent when these new forces are used. Applications of these new NN potentials do not lead to distortions in the cross section minimum of the higher energy elastic Nd scattering that were found in Ref. [18]. Also convergence with respect to the order of chiral expansion is improved when the new chiral forces are applied, as exemplified in Figure $2 \mathrm{C}$ for the analyzing power $A_{y}$. At low energies of the incoming neutron, theoretical predictions of standard NN potentials fail to explain the experimental data for $A_{y}$ (see Figure 2A). When instead of the standard forces the old chiral NN interactions with nonlocal momentum space regulator are used, the predictions for $A_{y}$ vary with the order of chiral expansion. In particular, the NLO results overestimate the $A_{y}$ data while $\mathrm{N}^{2} \mathrm{LO} \mathrm{NN}$ forces seem to provide quite a good description of them (see Figure $2 \mathrm{~B}$ ). However, when $\mathrm{N}^{3} \mathrm{LO} \mathrm{NN}$ chiral forces are used, a clear discrepancy between theory and data emerges in the region of $A_{y}$ maximum, which is similar to the one for the standard forces. Using a new procedure for estimating the theoretical truncation errors introduced in $[10,11]$, we show in Figure $2 \mathrm{C}$ theoretical uncertainties at different 
orders of chiral expansion for $A_{y}$ predictions with the improved potentials. With increasing order the uncertainties become very small and very precise predictions are provided at $\mathrm{N}^{3} \mathrm{LO}$ and $\mathrm{N}^{4} \mathrm{LO}$. It is interesting to note that the width of the uncertainty band at $\mathrm{N}^{2} \mathrm{LO}$ is of the same size as the discrepancy with respect to the data. At that order for the first time 3NF, which is neglected in the present calculations, appears. Therefore one can expect that including consistent $\mathrm{N}^{3} \mathrm{LO}$ chiral 3NF's will probably provide an explanation for $A_{y}$ puzzle.

That new procedure for estimating the theoretical truncation errors provides also evidence for missing $3 \mathrm{NF}$ effects at higher energy elastic Nd scattering, supporting thus the results found with the standard NN potentials. It remains to be seen whether consistent $3 \mathrm{NF}^{\prime}$ 's at $\mathrm{N}^{3} \mathrm{LO}$ will resolve higher energy discrepancies found in $\mathrm{Nd}$ elastic scattering.

\section{Summary}

Solving $3 \mathrm{~N}$ scattering exactly up to energies below the pion production threshold allows one to test the 3N Hamiltonian based on modern NN potentials and 3NF's. At the higher energies for some observables large 3NF effects are predicted when using models such as TM and Urbana IX. Some Nd elastic scattering cross section and polarization data support these predictions. In some other cases, however, defects of the (semi)phenomenological 3NF's are demonstrated. Relativistic effects are found to be small for the elastic scattering cross section and negligible for higher energy spin-observables. The discrepancies at high energies, which remain even when Urbana IX or TM 3NF's are included, point to the importance of short-range contributions to the $3 \mathrm{NF}$. Application of improved chiral NN interactions up to $\mathrm{N}^{4} \mathrm{LO}$ order of chiral expansion and estimation of theoretical uncertainties due to truncation of higher orders supports conclusions obtained with the standard NN potentials. Higher order chiral 3NF's comprise a number of shorter-range terms. It can be expected that an application of consistent chiral $\mathrm{NN}$ and $3 \mathrm{~N}$ forces will play an important role in understanding of elastic scattering and breakup reactions at higher energies.

\section{Acknowledgments}

This work was supported by the Polish National Science Center under Grant No. DEC-2013/10/M/ST2/00420. Some part of the work was performed within LENPIC collaboration. The numerical calculations have been performed on the supercomputer cluster of the JSC, Jülich, Germany. 


\section{References}

[1] Wiringa R. B. et al., Phys. Rev. C, 51 (1995) 38.

[2] Machleidt R., Phys. Rev. C, 63 (2001) 024001.

[3] Stoks V. G. J. et al., Phys. Rev. C, 49 (1994) 2950.

[4] Pudliner B. S. et al., Phys. Rev. C, 56 (1997) 1720.

[5] Coon S. A. et al., Nucl. Phys. A, 317 (1979) 242.

[6] Epelbaum E., Prog. Part. Nucl. Phys., 57 (2006) 654.

[7] Machleidt R. and Entem D. R., Phys. Rep., 503 (2011) 1.

[8] Epelbaum E. et al., Phys. Rev. C, 66 (2002) 064001.

[9] Bernard V. et al., Phys. Rev. C, 84 (2011) 054001.

[10] Epelbaum E. et al., Eur. Phys. J. A, 51 (2015) 53.

[11] Epelbaum E. et al., arXiv:1412.4623 [nucl-th].

[12] Glöckle W. et al., Phys. Rep., 274 (1996) 107.

[13] Witała H. et al., Phys. Rev. C, 63 (2001) 024007.

[14] Sekiguchi K. et al., Phys. Rev. C, 79 (2009) 054008.

[15] Stephan E. et al., Eur. Phys. Journal A, 42 (2009) 13.

[16] Witała H. et al., Phys. Rev. C, 71 (2005) 054001.

[17] Witała H. et al., Phys. Rev. C, 77 (2008) 034004.

[18] Witała H. et al., J. Phys. G, 41 (2014) 094011.

[19] Maeda Y. et al., Phys. Rev. C, 76 (2007) 014004.

[20] Coon S. A. and Han H. K., Few-Body Systems, 30 (2001) 131.

[21] Tornow W. et al., Phys. Rev. Lett., 49 (1982) 312. 